\title{
Cryptococcal-related Mortality Despite Fluconazole Preemptive Treatment in a Cryptococcal Antigen Screen-and-Treat Program
}

\author{
Rachel M. Wake, ${ }^{1,2}$ Nelesh P. Govender, ${ }^{1,3,4}$ Tanvier Omar, $^{5,6}$ Carolina Nel, ${ }^{5,6}$ Ahmad Haeri Mazanderani, ${ }^{7,8}$ Aaron S. Karat, ${ }^{9}$ Nazir A. Ismail, ${ }^{10}$
} Caroline T. Tiemessen, ${ }^{7,11}$ Joseph N. Jarvis, ${ }^{12,13,14}$ and Thomas S. Harrison ${ }^{2}$

${ }^{1}$ Centre for Healthcare-associated Infections, Antimicrobial Resistance and Mycoses, National Institute for Communicable Diseases, Johannesburg, South Africa; ${ }^{2}$ Institute of Infection \& Immunity, St George's University of London, United Kingdom; ${ }^{3}$ School of Pathology, University of the Witwatersrand, Johannesburg, ${ }^{4}$ Division of Medical Microbiology, University of Cape Town, ${ }^{5}$ Department of Anatomical Pathology, University of the Witwatersrand, ${ }^{6}$ Department of Pathology, National Health Laboratory Services, and ${ }^{7}$ Centre for HIV \& STIs, National Institute for Communicable Diseases, Johannesburg, and ${ }^{8}$ Department of Medical Virology, University of Pretoria, South Africa; ${ }^{9}$ Tuberculosis Centre, London School of Hygiene \& Tropical Medicine, United Kingdom; ${ }^{10}$ Centre for Tuberculosis, National Institute for Communicable Diseases, and ${ }^{11}$ Faculty of Health Sciences, University of the Witwatersrand, Johannesburg, South Africa; ${ }^{12}$ Department of Clinical Research, Faculty of Infectious and Tropical Diseases, London School of Hygiene and Tropical Medicine, United Kingdom; ${ }^{13}$ Botswana-UPenn Partnership, Gaborone; and ${ }^{14}$ Division of Infectious Diseases, Department of Medicine, Perelman School of Medicine, University of Pennsylvania, Philadelphia

\section{(See the Editorial Commentary by Rajasingham and Boulware on pages 1691-4.)}

Background. Cryptococcal antigen (CrAg) screening and treatment with preemptive fluconazole reduces the incidence of clinically evident cryptococcal meningitis in individuals living with advanced human immunodeficiency virus (HIV) disease. However, mortality remains higher in CrAg-positive than in CrAg-negative patients with similar CD4+ T-lymphocyte counts.

Methods. We conducted a cohort study to investigate causes of morbidity and mortality during 6 months of follow-up among asymptomatic CrAg-positive and CrAg-negative (ratio of 1:2) patients living with HIV with CD4 counts $<100$ cells/ $\mu \mathrm{L}$ attending 2 hospitals in Johannesburg, South Africa. When possible, minimally invasive autopsy (MIA) was performed on participants who died.

Results. Sixty-seven CrAg-positive and 134 CrAg-negative patients were enrolled. Death occurred in 17/67 (25\%) CrAgpositive and 12/134 (9\%) CrAg-negative participants (hazard ratio for death, adjusted for CD4 count, 3.0; 95\% confidence interval, 1.4-6.7; $P=.006)$. Cryptococcal disease was an immediate or contributing cause of death in 12/17 (71\%) CrAg-positive participants. Postmortem cryptococcal meningitis and pulmonary cryptococcosis were identified at MIA in all 4 CrAg-positive participants, 3 of whom had negative cerebrospinal fluid CrAg tests from lumbar punctures (LPs) at the time of CrAg screening.

Conclusions. Cryptococcal disease was an important cause of mortality among asymptomatic CrAg-positive participants despite LPs to identify and treat those with subclinical cryptococcal meningitis and preemptive fluconazole for those without meningitis. Thorough investigation for cryptococcal disease with LPs and blood cultures, prompt ART initiation, and more intensive antifungals may reduce mortality among asymptomatic CrAg-positive patients identified through screening.

Keywords. cryptococcus; acquired immunodeficiency syndrome; AIDS-related opportunistic infections; autopsy; cryptococcal meningitis.

Cryptococcal disease is a leading cause of AIDS-related death in sub-Saharan Africa [1]. Although cryptococcal antigen (CrAg) screening and preemptive treatment with fluconazole reduces the incidence of cryptococcal meningitis [2-5], individuals with cryptococcal antigenemia treated with fluconazole remain at higher risk of death than individuals without cryptococcal antigenemia with similar CD4+ T-lymphocyte (CD4) counts [2-4].

Received 22 January 2019; editorial decision 25 April 2019; accepted 7 June 2019; published online June 8, 2019

Correspondence: R. M. Wake, Centre for Healthcare-associated Infections, Antimicrobial Resistance and Mycoses, National Institute for Communicable Diseases, NICD, 1 Modderfontein Road, Johannesburg, 2131, South Africa (rmwake@gmail.com).

\section{Clinical Infectious Diseases ${ }^{\circledR} \quad$ 2020;70(8):1683-90}

(C) The Author(s) 2019. Published by Oxford University Press for the Infectious Diseases Society of America. All rights reserved. For permissions, e-mail: journals.permissions@oup.com. DOI: $10.1093 /$ cid/ciz485
CrAg screen-and-treat strategies have been implemented in several countries where cryptococcal meningitis is a common opportunistic infection [6]. In South Africa, blood samples with CD4 counts $<100$ cells/ $\mu \mathrm{L}$ are screened at central laboratories using the Immuno-mycologics (IMMY, Norman, OK) $\mathrm{CrAg}$ lateral flow assay (LFA). South African national guidelines stipulate that CrAg-positive patients are assessed for symptoms or signs of meningitis and, if present, investigated for cryptococcal meningitis with a lumbar puncture (LP). Asymptomatic patients are offered LPs if available at the screening site or nearby facility and started on preemptive fluconazole ( $800 \mathrm{mg}$ daily for 2 weeks, followed by $400 \mathrm{mg}$ daily for 2 months, and then $200 \mathrm{mg}$ daily pending immune reconstitution) if cryptococcal meningitis is excluded or if LP is not performed. Antiretroviral therapy (ART) is delayed for 2 weeks in asymptomatic CrAg-positive patients and for 4-6 weeks if cryptococcal meningitis is diagnosed [7]. 
The causes of excess mortality among CrAg-positive compared to CrAg-negative patients are as yet unclear. Previous studies have not fully investigated causes of death; no autopsies were performed $[2-4,8,9]$. Since very few $(<5 \%)$ CrAg-positive patients were known to develop cryptococcal meningitis following screening and treatment $[3,4,8]$, noncryptococcal causes were suspected.

We investigated morbidity and mortality during 6 months following screening among CrAg-positive and CrAg-negative adults living with human immunodeficiency virus (HIV) in Johannesburg, South Africa. Causes of death were investigated, including by minimally invasive autopsy (MIA).

\section{METHODS}

Routine CrAg screening was performed on all individuals living with HIV with CD 4 counts $<100$ cells/ $\mu \mathrm{L}$ at Helen Joseph and Tambo Memorial hospitals, Johannesburg. HIV-seropositive adults (aged $\geq 18$ years) with CD 4 counts $<100$ cells $/ \mu \mathrm{L}$ who were CrAg-positive were sequentially invited to participate when they attended the hospitals' HIV clinics or wards for results between June 2015 and October 2017. CrAg-negative patients with similar CD4 counts $( \pm 10$ cells/ $\mu \mathrm{L})$ were concurrently recruited at a ratio of 2:1. Patients were excluded if they had symptoms or signs of cryptococcal meningitis (ie, severe headache or reduced level of consciousness) at any time since screening or if they were receiving antifungal treatment for previous cryptococcal meningitis. Written informed consent for participation and for MIA in the event of death was requested. The study was granted ethics approval by the University of the Witwatersrand and the London School of Hygiene \& Tropical Medicine.

Training on the clinical algorithm for management of CrAgpositive patients [7] was provided to health workers at study sites by the principal investigator (R. M. W.). Study participants were assessed and managed by their usual clinicians following CrAg screening. A study nurse collected clinical data including symptoms, examination findings, medical diagnoses, and medication at face-to-face consultations, from clinical records at enrollment, and on 3 subsequent routine visits over 6 months. Time to treatment initiation was measured from the day that screening blood arrived in the laboratory. Adherence was assessed at participant visits and by review of pharmacy records. At enrollment, participants provided blood samples for prolonged (21 days) fungal culture and C-reactive protein (CRP) testing; pre- and/or postinduction sputum for fungal culture and tuberculosis (TB) testing (auramine staining and microscopy, liquid culture [BACTEC MGIT, Becton Dickinson, Franklin Lakes, NJ] and molecular testing [Xpert MTB/RIF, Cepheid, Sunnyvale, CA]); and urine for lipoarabinomannan (Determine TB LAM, Alere, Waltham, MA), Histoplasma antigen enzyme immunoassay (IMMY), and Xpert MTB/RIF Ultra testing. Sputum and blood culture results were provided to the participant's clinician if clinically significant. Further assessment and TB treatment were recommended if the urine TB LAM test was positive. Participants were contacted by telephone every two weeks to discuss any new medical problems. If no contact was made, contact with a friend or relative named by the participant was attempted.

Cryptococcal disease was defined as any participant with cryptococcal meningitis (Cryptococcus identified by cerebrospinal fluid [CSF] microscopy with India ink, fungal culture, and/or CrAg testing), cryptococcemia (Cryptococcus cultured from blood), or pulmonary cryptococcosis (Cryptococcus cultured from sputum). Asymptomatic cryptococcal antigenemia alone was not categorized as cryptococcal disease for the purposes of this study.

In the event of death, information regarding recent symptoms and hospital admissions was obtained from the participant's closest relative and clinical records. Permission was requested from the family to perform MIA if informed consent had been provided. MIAs were performed by the principal investigator (R. M. W.) with the assistance of a study nurse. An initial external examination of the body was performed, and organs were located using external anatomical landmarks or ultrasound. Skin was cleaned with iodine and half-centimeter incisions made at biopsy sites. Standardized multiple core biopsy samples were obtained from lungs, liver, spleen, and kidneys using a 14-gauge core biopsy needle. CSF from the cisterna magna (suboccipital approach) and blood from the subclavian vein were aspirated using 18-gauge needles and syringes. Bronchoalveolar lavage (BAL) was performed using normal saline delivered through a nasogastric tube into the bronchi through a tracheal incision. If abnormalities were clinically detected, aspirates of pleural and pericardial fluid and punch biopsies of any skin lesions (excluding on the face or neck) were taken.

Samples underwent microbiological testing at the National Institute for Communicable Disease and histological analysis at the University of the Witwatersrand. Investigators who performed analyses were not aware of the participants' antemortem CrAg status.

Tissue cores were preserved in formalin, embedded in paraffin, and stained with hematoxylin-eosin. Additional stains to assess for the presence of acid-fast bacilli (Ziehl-Neelsen), bacteria (Gram stain), and fungi (Grocott's methenamine silver) were performed if any features of inflammation were seen. Cytomegalovirus immunoperoxidase staining was performed when characteristic viral inclusions were noted.

Tissue cores, CSF, BAL, and pericardial fluid underwent bacterial, fungal, and mycobacterial cultures; bacterial and viral multiplex polymerase chain reaction; and Xpert MTB/ RIF testing. Plasma, CSF, and BAL fluid were tested using the IMMY CrAg LFA.

Immediate and contributing causes of death were attributed by a panel of 6 of the authors (infectious diseases physicians and pathologists) at the University of the Witwatersrand Department 
of Anatomical Pathology, which has extensive experience conducting MIA studies [10-12]. Procedures were similar to those described in previous MIA studies [10, 11]: organisms isolated on culture from postmortem samples were considered to be pathological if the same organism was cultured from a premortem sample, if there was clinical evidence of infection with the organism, or if histology showed consistent changes within the affected organ. Diagnoses were "possible," "probable," or "likely" according to the data available and categorized using the Coding of Causes of Death in HIV protocol [13].

Baseline demographic and clinical variables were compared using $\chi^{2}$, Fisher exact, or Mann-Whitney $U$ tests, as appropriate. Clinical outcomes during follow-up were compared using rate ratios with 95\% confidence intervals (CIs). The effect of $\mathrm{CrAg}$ status on mortality, adjusted for baseline CD4 count, was estimated using Cox proportional hazards regression analysis.
Other explanatory variables were evaluated as risk factors for death, including age, sex, baseline CD4 count (dichotomized at a threshold of $<50$ cells $/ \mu \mathrm{L}$ ), ART status, low body mass index (BMI; $<18 \mathrm{~kg} / \mathrm{m}^{2}$ ), severe anemia (hemoglobin $\leq 8 \mathrm{~g} / \mathrm{dL}$ ), and active TB (diagnosed at enrollment, within the past 6 months, or if they remained on treatment). A sensitivity analysis was performed assuming that any participants lost to follow-up had died. A sample size of $63 \mathrm{CrAg}$-positive participants was required to detect, with $80 \%$ power and $5 \%$ significance level, a mortality difference of $18 \%$, consistent with previous studies [2-4].

\section{RESULTS}

Of 7149 participants screened at the sites during the study period, 604 (8.4\%) were CrAg-positive. Of these, 67 (11\%) CrAgpositive and 134 (2\%) CrAg-negative patients were enrolled in the study (Figure 1, Supplementary Table 3). There were

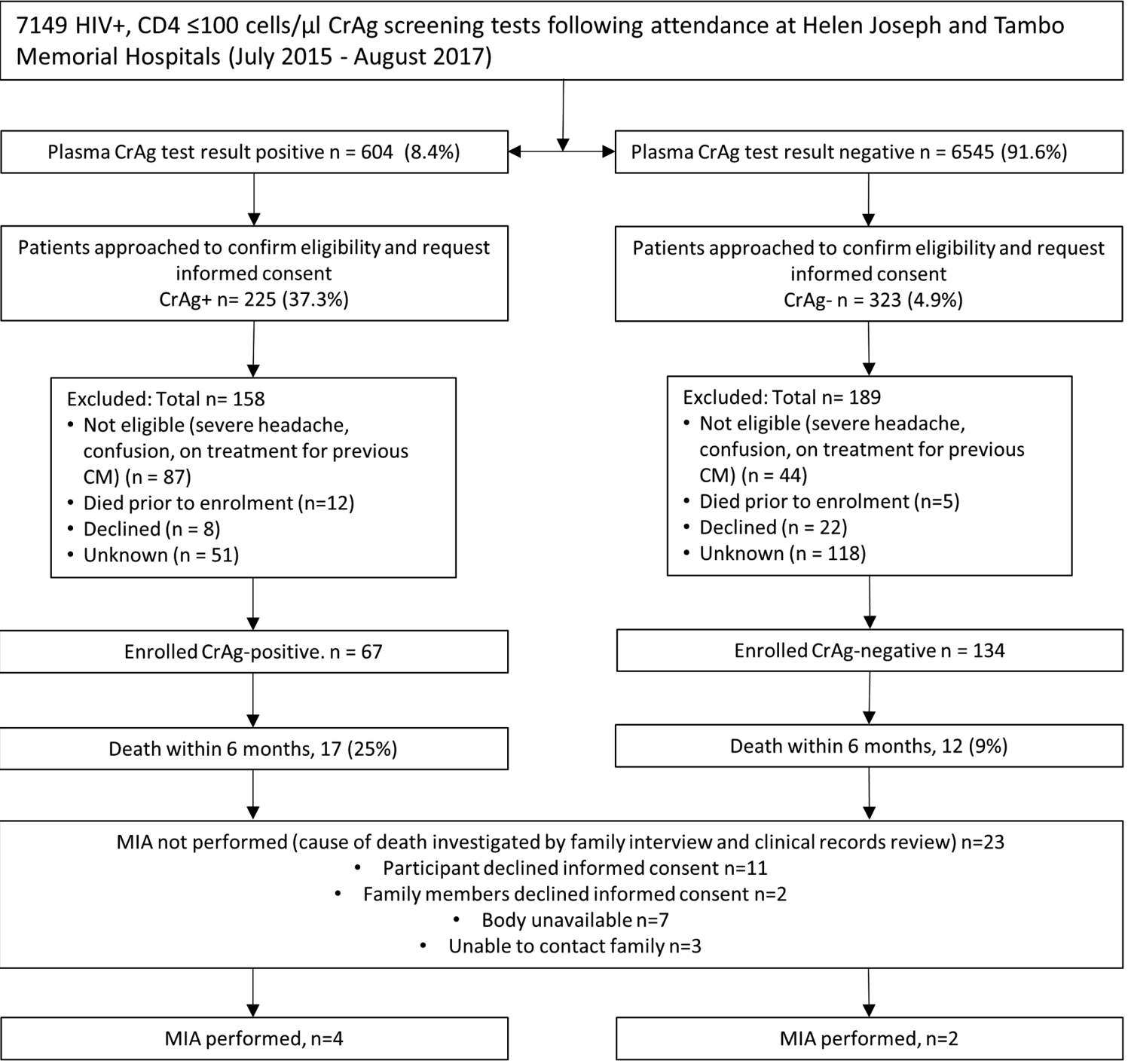

Figure 1. Flow diagram showing patients included and excluded in the prospective cohort study. Abbreviations: CD4, CD4 T-lymphocyte count; CM, cryptococcal meningitis; CrAg, cryptococcal antigen; HIV, human immunodeficiency virus; MIA, minimally invasive autopsy. 
no differences in demographic characteristics, prior or prevalent AIDS-defining illnesses (including TB and histoplasmosis following screening at enrollment), other infectious or noninfectious medical conditions, CRP, BMI, or hemoglobin at baseline between $\mathrm{CrAg}$-positive and $\mathrm{CrAg}$-negative participants (Table 1). CrAg-positive participants who had lower CD4 counts (27 cells/ $\mu \mathrm{L}$; interquartile range [IQR], $7-40$ vs 41 cells/ $\mu \mathrm{L} ; \mathrm{IQR}$, $16-64 ; P=.002)$, were more likely to have already started ART (24\% vs $10 \%, P=.01$ ) and prophylactic co-trimoxazole $(55 \%$ vs $24 \%, P<.001)$ at the time of enrollment and less likely to be South African ( $42 \%$ vs $25 \%, P=.01$ ). Follow-up for 6 months or to time of death if earlier was completed for 193/201 (96\%) of participants.

\section{Evidence for Cryptococcal Disease at Baseline}

Cryptococcal disease was found in 17/67 (25\%; 95\% CI, 16\%37\%) asymptomatic CrAg-positive participants: subclinical cryptococcal meningitis in 11/57 (19\%; 95\% CI, 10\%-32\%) who had LPs, cryptococcemia in 11/67 (16\%) who had prolonged fungal blood cultures ( 4 had no evidence of cryptococcal meningitis on LP), and pulmonary cryptococcosis in 2/32 (7\%) who had sputum fungal cultures ( 1 had a negative LP). Plasma CrAg titers were higher among those who had cryptococcal disease than those with asymptomatic cryptococcal antigenemia alone (median, 5120; IQR, 160-10, 240 vs 10; IQR, 5-80; $P<.001$ ) and in those with subclinical cryptococcal meningitis than those who had negative LPs (median, 10, 240; IQR, 1280-81 920 vs 15; IQR, 5-160; $P<.001)$. No CrAg-negative participants had cryptococcal disease at baseline.

\section{Treatment Received}

Antifungal treatment in accordance with guidelines at the time [7] (fluconazole $800 \mathrm{mg}$ daily for those without and amphotericin B $1 \mathrm{mg} / \mathrm{kg}$ daily with fluconazole $800 \mathrm{mg}$ daily for those with cryptococcal meningitis) was commenced for 62/67 (93\%) participants at a median of 7 (IQR, 3-12) days following

Table 1. Baseline Characteristics of the Cryptococcal Antigen (CrAg)-positive and CrAg-negative Patients

\begin{tabular}{|c|c|c|c|}
\hline Characteristic & $\begin{array}{l}\text { CrAg-positive } \\
\quad(n=67)\end{array}$ & $\begin{array}{l}\text { CrAg-negative } \\
\quad(n=134)\end{array}$ & $P$ Value \\
\hline Age, median (IQR), y & $39(32-47)$ & $39(33-48)$ & $.63^{\mathrm{a}}$ \\
\hline Female (\%) & $37(55)$ & $62(46)$ & .23 \\
\hline Self-assigned black race (\%) & 66 (99) & $128(96)$ & .4 \\
\hline Born outside of South Africa ${ }^{b}(\%)$ & $28(42)$ & $33(25)$ & .01 \\
\hline Non-Southern Africa Development Community (\%) & $3(5)$ & $3(2)$ & .4 \\
\hline CD4 T-lymphocyte count cells/uL (median, IQR) & $27(7-40)$ & $41(16-64)$ & $.002^{\mathrm{a}}$ \\
\hline$<50(\%)$ & $55(82)$ & $80(60)$ & .001 \\
\hline Body mass index $<18 \mathrm{~kg} / \mathrm{m}^{2}(\%)^{\mathrm{c}}$ & $9(20)$ & 20(18) & $1.0^{d}$ \\
\hline Hemoglobin $\leq 8 \mathrm{~g} / \mathrm{dL}(\%)^{\mathrm{e}}$ & $5(9)$ & $13(11)$ & $.8^{d}$ \\
\hline C-reactive protein, mg/L (median, IQR) ${ }^{f}$ & $15(3-49)$ & $22(4-56)$ & .4 \\
\hline Active TB at enrollment ${ }^{g}(\%)$ & $19(28)$ & $33(25)$ & 6 \\
\hline Previous TB ${ }^{h}(\%)$ & $11(16)$ & $14(10)$ & $.3^{d}$ \\
\hline Other previous or current ADI (\%) & $11(16)$ & $20(15)$ &.$^{d}$ \\
\hline Histoplasmosis & $3(5)$ & $3(2)$ & $.4^{d}$ \\
\hline Other (non-ADI) medical conditions (\%) & $30(45)$ & $57(43)$ & .8 \\
\hline Infectious disease (\%) & $18(27)$ & $39(29)$ & .7 \\
\hline Hepatitis $B^{j}$ & $6(17)$ & $11(13)$ & $.6^{d}$ \\
\hline Immunocompromising (\%) & $2(3)$ & $4(3)$ & $1.0^{\mathrm{d}}$ \\
\hline Taking antiretroviral therapy (>1 day) (\%) & $16(24)$ & $13(10)$ & $.01^{d}$ \\
\hline Taking co-trimoxazole (\%) & $37(55)$ & $32(24)$ & $<.001$ \\
\hline Taking other antibiotics (\%) & $22(33)$ & $37(28)$ & .4 \\
\hline
\end{tabular}

Pearson $\chi^{2}$ test used unless indicated.

Abbreviations: ADI, AIDS-defining illness; CrAg, cryptococcal antigen; IQR, interquartile range; TB, tuberculosis.

${ }^{a}$ Mann-Whitney $U$ test.

${ }^{b}$ Data missing on birthplace for 1 patient.

'Data missing on BMI for 20 CrAg-positive and 23 CrAg-negative patients.

${ }^{\mathrm{d}}$ Fisher exact test.

${ }^{e}$ Data missing on hemoglobin for $12 \mathrm{CrAg}$-positive and $15 \mathrm{CrAg}$-negative patients.

${ }^{\mathrm{f}}$ Data missing on $\mathrm{C}$-reactive protein for $5 \mathrm{CrAg}$-positive and $5 \mathrm{CrAg}$-negative patients.

${ }^{9}$ Active TB includes those diagnosed with TB within the past 6 months and/or on current TB treatment at enrollment (CrAg-positive, $n=12,18 \% ;$ CrAg-negative, $\left.n=13,10 \%\right)$, including those diagnosed on the day of enrollment (CrAg-positive, $n=7,10 \%$; CrAg-negative, $n=20,15 \%$ ). Diagnoses of TB were made clinically or on the basis of routine laboratory tests, or screening performed as part of the study.

hPrevious TB includes those who were diagnosed more than 6 months prior to enrollment and who do not remain on TB treatment.

'Data missing on histoplasmosis screening (urine enzyme immunoassay for $1 \mathrm{CrAg}$-positive and $7 \mathrm{CrAg}$-negative patients;

'Data missing on hepatitis B for 32 CrAg-positive and 50 CrAg-negative patients. 
a positive $\mathrm{CrAg}$ test. Good adherence to the recommended antifungal regimen was reported for $40 / 57$ (70\%) during the 6 months following $\mathrm{CrAg}$ screening. Three $\mathrm{CrAg}$-positive participants had no record of receiving antifungals; 1 was lost to follow-up, 2 survived.

Fifty-three of 67 (79\%) CrAg-positive and 128 (96\%) CrAgnegative participants commenced ART during the study period (rate ratio [RR], 0.97; 95\% CI, 0.71-1.34; $P=.9$ ). The median delay between CD4 count and ART initiation was 36 (IQR, 26-45) days for CrAg-positive participants and 17 (IQR, 7-32) days for CrAg-negative participants $(P<.001)$. Among CrAgpositive participants, median delay to ART initiation was 21 (IQR, 8-57) days for participants with cryptococcal meningitis and 36 (IQR, 31-49) days for those without cryptococcal meningitis.

The proportions of CrAg-positive and CrAg-negative participants who took any antibiotic during the study period were similar $(25 / 67,37 \%$ vs $42 / 134,31 \%$; $P=.4)$.

\section{Clinical Outcomes}

There were no significant differences between CrAg-positive and CrAg-negative participants in the proportion with undetectable HIV-1 viral loads within 6 months, BMI change, and incidence of TB or other infectious or noninfectious medical conditions during follow-up. Other AIDS-defining illnesses (not cryptococcosis or TB) developed in 8 (12\%) CrAg-positive and 7 (5\%) CrAg-negative participants (RR, 2.69; 95\% CI, 0.98 7.42; $P=.05$; Table 2).

\section{Cryptococcal Disease During Follow-up}

One CrAg-positive participant who was diagnosed and treated for subclinical cryptococcal meningitis at enrollment developed a second episode 5 months later, despite reported adherence to fluconazole maintenance therapy and ART. Four of 11 (36\%) participants with baseline subclinical cryptococcal meningitis died during follow-up; 3 following treatment with amphotericin $B$ and fluconazole $(13,14$, and 25 days after LP), 1 participant received fluconazole only and died 3 months later. Two of these participants also had cryptococcemia at baseline, 1 had cryptococcal pneumonia identified at MIA.

Thirteen of 46 (28\%) CrAg-positive participants died following negative LPs (none had cryptococcemia, 1 had growth of Cryptococcus neoformans on sputum at baseline). Eight of these deaths were cryptococcal-related: 4 cases of cryptococcal meningitis (3 identified at MIA, 1 clinically diagnosed prior to death, at least 2 had good adherence to fluconazole), 1 case of pulmonary cryptococcal immune reconstitution inflammatory syndrome, 1 case of cryptococcal pneumonia, and 2 deaths in participants who defaulted preemptive fluconazole treatment (immediate cause of death unknown).

No cryptococcal disease was identified in any CrAg-negative participants during follow-up.

\section{Mortality}

Death occurred in $14 \%$ (29/201, rate 0.4 deaths/person-year [d/PY]) of all participants within 6 months; $25 \%$ (17/67, $0.7 \mathrm{~d} /$ PY) CrAg-positive and 9\% (12/134, $0.2 \mathrm{~d} / \mathrm{PY})$ CrAg-negative

Table 2. Clinical Outcomes During Follow-up in Cryptococcal Antigen (CrAg)-positive and CrAg-negative Patients

\begin{tabular}{|c|c|c|c|c|c|}
\hline Clinical Outcome & $\begin{array}{c}\text { Total } \\
(N=201)\end{array}$ & $\begin{array}{l}\text { CrAg-positive } \\
\quad(n=67)\end{array}$ & $\begin{array}{l}\text { CrAg-negative } \\
\quad(n=134)\end{array}$ & $\begin{array}{l}\text { Rate Ratio in CrAg-positive vs } \\
\text { CrAg-negative }(95 \% \mathrm{Cl})\end{array}$ & PValue \\
\hline New ADI, n (rate/1000 PY, 95\% CI) & $24(0.8,0.5-1.2)$ & $10(1.1,0.6-2.1)$ & $14(0.7,0.4-1.1)$ & $1.68(.75-3.78)$ & .2 \\
\hline Tuberculosis, ${ }^{a}$ n (rate/1000 PY, 95\% Cl) & $10(0.3,0.2-0.6)$ & $2(0.2,0.1-1.0)$ & $8(0.4,0.2-0.8)$ & $0.54(.12-2.66)$ & .5 \\
\hline Other ${ }^{\mathrm{b}}$ ADIs, n (rate/1000 PY, 95\% CI) & $15(0.5,0.3-0.8)$ & $8(0.9,0.4-1.8)$ & $7(0.3,0.2-0.7)$ & $2.69(.98-7.42)$ & .05 \\
\hline $\begin{array}{l}\text { New (non-ADI) medical condition, } \\
\text { n (rate/1000 PY) }\end{array}$ & $51(1.7,1.3-2.2)$ & $21(2.3,1.5-3.6)$ & $30(1.4,1.0-2.0)$ & $1.65(.94-2.88)$ & .08 \\
\hline Infectious disease, n (rate/1000 PY) & $25(0.8,0.6-1.2)$ & $9(1.0,0.5-1.9)$ & $16(0.8,0.5-1.2)$ & $1.32(.59-3.00)$ & .5 \\
\hline $\begin{array}{l}\text { Body mass index change, }{ }^{\mathrm{C}} \mathrm{kg} / \mathrm{month} \\
\text { (median, IQR) }\end{array}$ & $0.24(0-0.24)$ & $0.21(-0.02$ to 0.35$)$ & $0.24(0.06-0.49)$ & $\ldots$ & $3^{d}$ \\
\hline $\begin{array}{l}\text { Undetectable viral load } \\
\qquad(\leq 50 \text { copies } / \mathrm{mL})(\%)\end{array}$ & $31(27)$ & $7(28)$ & $24(27)$ & $\ldots$ & $1.0^{f}$ \\
\hline Deaths within 6 months (\%) & $29(14)$ & $17(25)$ & $12(9)$ & $3.28(1.57-6.87)$ & .002 \\
\hline $\begin{array}{l}\text { Median (IQR) days from CD4 to death } \\
(n=29)\end{array}$ & $53(20-74)$ & $26(14-52)$ & 70 (37-95) & & .02 \\
\hline
\end{tabular}

Pearson $\chi^{2}$ test used unless indicated.

Abbreviations: ADI, AIDS-defining illness; $\mathrm{Cl}$, confidence interval; CrAg, cryptococcal antigen; IQR, interquartile range; PY, person-years.

${ }^{a}$ Of those not diagnosed at enrollment: $60 \mathrm{CrAg}$-positive and $114 \mathrm{CrAg}$-negative patients. TB diagnosed during follow-up, not including at baseline.

${ }^{b} \mathrm{ADIs}$ other than cryptococcosis or tuberculosis (TB). CrAg-positive patients developed esophageal candidiasis $(n=2)$; cytomegalovirus $(\mathrm{CMV})$ other than liver, spleen, nodes $(\mathrm{n}=1)$; CMV retinitis $(n=2)$; herpes simplex (chronic ulcers) $(n=3)$; disseminated histoplasmosis $(n=1)$; Kaposi sarcoma $(n=1)$; Mycobacterium avium complex $(n=1)$; progressive multifocal leukoencephalopathy $(n=1)$; wasting syndrome $(n=1)$. CrAg-negative patients developed esophageal candidiasis $(n=2) ; C M V(n=2) ;$ Kaposi sarcoma $(n=4) ;$ Pneumocystis jirovecii pneumonia $(n=2)$; recurrent pneumonia $(n=2)$; wasting syndrome $(n=1)$.

${ }^{c} \mathrm{Of}$ those with subsequent weight measurements: $38 \mathrm{CrAg}$-positive and $90 \mathrm{CrAg}$-negative patients.

${ }^{\mathrm{d}}$ Fisher exact test.

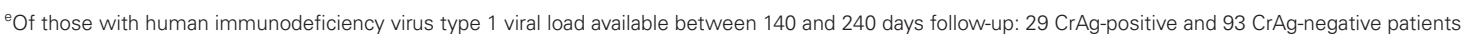

${ }^{\dagger}$ Mann-Whitney U test. 


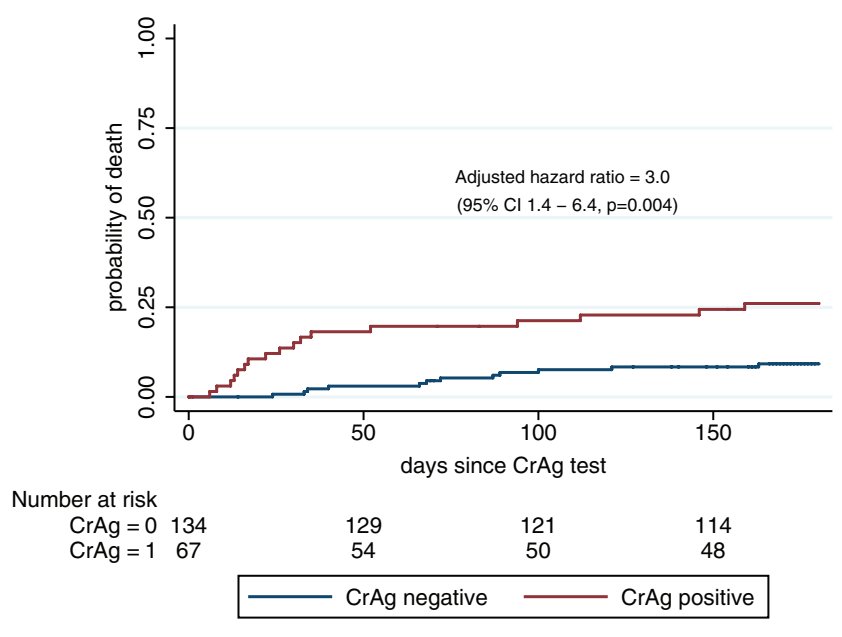

Figure 2. Kaplan-Meier curve showing mortality estimates in CrAg-positive and CrAg-negative patients within 6 months, adjusted for baseline CD4 T-lymphocyte count. Abbreviations: $\mathrm{Cl}$, confidence interval; $\mathrm{CrAg}$, cryptococcal antigen.

(Table 2, Figure 2). Participants with cryptococcal antigenemia had 3.3 times increased risk of death $(95 \% \mathrm{CI}, 1.6-7.0 ; P<.001)$ compared to CrAg-negative participants. This association remained significant when adjusted for baseline $\mathrm{CD} 4$ count $(<50$ cells $/ \mu \mathrm{L}$; hazard ratio $[\mathrm{HR}], 3.0 ; 95 \% \mathrm{CI}, 1.4-6.4 ; P=.004$ ) and if all those lost to follow-up were assumed to have died (HR, 2.4; 95\% CI, 1.3-4.6; $P=.008$ ). Of other baseline variables assessed, only active $\mathrm{TB}$ at the time of enrollment was associated with mortality (HR, 2.6; 95\% CI, 1.2-5.4; $P=.01$ ). Among CrAg-positive participants, death was associated with having a higher plasma CrAg titer (HR, 3.5 if titer $>160$ vs $\leq 160 ; 95 \%$ CI, $1.4-9.2 ; P=.009$ ) but not with baseline cryptococcal disease. Time from CrAg test to death was a median 26 (IQR, 14-52) days in CrAg-positive and 70 (IQR, 37-95) days in CrAg-negative participants $(P=.02)$.

\section{MIA Results}

MIAs were performed on $4 \mathrm{CrAg}$-positive and $2 \mathrm{CrAg}$-negative participants (Supplementary Table 2 and Figure 1). Postmortem cryptococcal meningitis was diagnosed in all 4 CrAg-positive participants by CSF CrAg test (plus culture for 1). Three of the participants had had negative LPs at the time of CrAg screening ( 7 , 25 , and 32 days prior to death); all became confused prior to death. The other participant died following treatment for subclinical cryptococcal meningitis. Cryptococcal pneumonia was identified histologically in lung tissue at MIA. BAL CrAg tests were positive in all 4 CrAg-positive participants. Although multiple other pathologies were identified from autopsy samples, there was no evidence of cryptococcal disease in either CrAg-negative participant.

\section{Causes of Death}

Cryptococcosis was an immediate $(n=5)$ or contributing $(\mathrm{n}=7)$ cause of death in $12 / 17$ (71\%) CrAg-positive participants (8/12, 67\% had cryptococcal meningitis) and no CrAg-negative participants. Of all 53 causes of death attributed (Supplementary
Table 1), cryptococcosis $(12,23 \%)$ was most common, followed by sepsis $(11,21 \%)$ and TB $(8,15 \%)$.

\section{DISCUSSION}

Cryptococcal antigenemia was a strong and independent predictor of mortality among adults living with HIV without severe headache or reduced consciousness at the time of $\mathrm{CrAg}$ screening. This is consistent with previous studies that have found an increased mortality risk, irrespective of CD4 cell count and despite preemptive fluconazole treatment for CrAgpositive patients [2-4]. We found clinical and pathological evidence of cryptococcosis as a significant cause of morbidity and mortality among CrAg-positive participants; one-quarter had cryptococcal disease at the time of $\mathrm{CrAg}$ screening (19\% had subclinical cryptococcal meningitis), 4 participants developed cryptococcal meningitis following negative baseline LPs, and more than two-thirds of deaths were attributed to cryptococcosis as an immediate or contributing cause.

Our findings emphasize the need to thoroughly investigate asymptomatic CrAg-positive patients for cryptococcal disease with LPs and blood cultures. However, we found that several cryptococcal-related deaths occurred despite the exclusion of cryptococcal disease at the time of screening. This indicates that fluconazole monotherapy, which is known to be suboptimal treatment for cryptococcal meningitis [14-17], is also inadequate for preventing deaths among CrAg-positive patients; enhanced treatment strategies are needed.

One approach would be to use adjunctive antifungal treatment for all CrAg-positive patients or for those with higher blood CrAg titers, identified by quantitative CrAg assays [18, 19]. We found blood CrAg titers of $>160$ to be predictive of death, consistent with previous studies [5, 20, 21]. Adjunctive treatment options include flucytosine (shown to be effective for treating cryptococcal meningitis in the Advancing Cryptococcal meningitis Treatment for Africa (ACTA) trial [22]) or a single dose of $10 \mathrm{mg} / \mathrm{kg}$ liposomal amphotericin (effective fungicidal activity in CSF [23] and currently in a phase 3 trial for treating cryptococcal meningitis [24]). Both treatments are safe and feasible to administer in outpatient settings; randomized, controlled trials are required to establish if they would reduce mortality in asymptomatic CrAg-positive patients.

Delayed ART commencement may have contributed to increased mortality in CrAg-positive participants. Delay was longer in participants without subclinical cryptococcal meningitis, despite fluconazole being started relatively promptly after CrAg screening. Although point-of-care CrAg testing might improve linkage to ART [25-29], continued health worker education is imperative to ensure CrAg-positive patients initiate ART after the recommended 14 days of fluconazole $800 \mathrm{mg}$ daily.

Previous studies have suggested that excess mortality among CrAg-positive patients might be explained by increased 
susceptibility to other infections such as TB $[2,3,30]$. Despite enhanced screening for TB at enrollment, we found no evidence of an association between cryptococcal antigenemia and TB. CrAg-positive patients were more likely to develop other AIDS-defining illnesses, and it is likely that these contributed to increased mortality.

The study was limited by several factors. Despite attempts to match CD4 counts $( \pm 10$ cells $/ \mu \mathrm{L})$, convenience sampling resulted in the enrollment of CrAg-negative patients with higher CD4 counts who were more likely to be ART-naive. It is notable, however, that CrAg-positive participants remained around three times more likely to die than CrAg-negative participants when adjusted for baseline CD4 cell count. Although the sample size was sufficient to detect a mortality difference, the relatively small cohort did not allow adjustment for more than one confounder simultaneously.

The study was also limited by varying levels of clinical information available for participants during follow-up and at the time of death; it was only possible to perform MIAs on a small proportion of those who died. MIAs identified several pathologies that were not diagnosed prior to death, including cryptococcal meningitis and pulmonary cryptococcosis, using $\mathrm{CSF} / \mathrm{BAL} \mathrm{CrAg}$ tests. It is possible that $\mathrm{CrAg}$ detection in postmortem samples might reflect contamination or leakage from blood. However, it is pertinent that all participants who had CrAg-positive CSF at MIA developed confusion prior to death. CSF CrAg testing is a standard autopsy procedure [31] and has been used in similar studies $[10,11]$. Furthermore, previous autopsy and BAL studies have found pulmonary cryptococcosis to be underdiagnosed among patients living with HIV [32-35].

This study provides valuable insight into the causes of excess mortality in CrAg-positive compared to CrAg-negative patients, with cryptococcosis remaining a leading cause despite preemptive fluconazole. Prompt initiation of ART and thorough screening for baseline cryptococcal disease are recommended in CrAg-positive patients. However, to fully realize the potential of CrAg screen-and-treat programs for reducing cryptococcalrelated mortality, adjunctive antifungal treatment should be considered.

\section{Supplementary Data}

Supplementary materials are available at Clinical Infectious Diseases online. Consisting of data provided by the authors to benefit the reader, the posted materials are not copyedited and are the sole responsibility of the authors, so questions or comments should be addressed to the corresponding author.

\section{Notes}

Acknowledgments. The authors thank the patients and their families who contributed to this study, particularly in giving consent for minimally invasive autopsies to take place, and the staff of mortuaries, clinics, hospitals, and laboratories for their assistance. Data collection was done by Neo Legare, Matshediso Mkhwenezi, and Siphiwe Kutta. Sample analysis was done by Tracey Shabangu, Ernest Tsotetsi, Serisha Naicker, Tsidido Maphanga, Shaheed Omar, Andries Dreyer, Anne Von Gottberg, Florette
Treurnicht, Nicole Wolter, Linda De Gouveia, Orienka Hellferscee, Ruth Mpembe, and Kedibone Ndlangisa.

Disclaimer. The content is solely the responsibility of the authors and does not necessarily represent the official views of the National Institutes of Health (NIH).

Financial support. This work was supported by the South African Medical Research Council (self-initiated research grant awarded to T. O.). R. M. W. received support from the National Institute for Health Research (ACF-2015-16-003), the St George's Hospital Research Charity, the Sir Ratanji Dalal Research Scholarship, and the Meningitis Research Foundation (1604.0). This work is in part supported by the South African Research Chairs Initiative of the Department of Science and Technology and National Research Foundation of South Africa (awarded to C. T. T.). N. P. G. was partly supported by the National Institute of Allergy and Infectious Diseases of the NIH, under award R01AI118511, and the Centers for Disease Control and Prevention. A. S. K. reports grants from the Bill and Melinda Gates Foundation.

Potential conflicts of interest. J. N. J. has received grants from Gilead Sciences, European \& Developing Countries Clinical Trials Partnership, Wellcome Trust, and Medical Research Council UK. T. S. H. has received grants and personal fees from Gilead Sciences and personal fees from Viamet and Pfizer. All other authors reported no potential conflicts. All authors have submitted the ICMJE Form for Disclosure of Potential Conflicts of Interest. Conflicts that the editors consider relevant to the content of the manuscript have been disclosed.

\section{References}

1. Rajasingham R, Smith RM, Park BJ, et al. Global burden of disease of HIVassociated cryptococcal meningitis: an updated analysis. Lancet Infect Dis 2017; 17:873-81.

2. Mfinanga S, Chanda D, Kivuyo SL, et al; REMSTART Trial Team. Cryptococcal meningitis screening and community-based early adherence support in people with advanced HIV infection starting antiretroviral therapy in Tanzania and Zambia: an open-label, randomised controlled trial. Lancet 2015; 385:2173-82.

3. Longley N, Jarvis JN, Meintjes G, et al. Cryptococcal antigen screening in patients initiating ART in South Africa: a prospective cohort study. Clin Infect Dis 2016; 62:581-7.

4. Pac L, Horwitz MM, Namutebi AM, et al. Implementation and operational research: integrated pre-antiretroviral therapy screening and treatment for tuberculosis and cryptococcal antigenemia. J Acquir Immune Defic Syndr 2015; 68:e69-76.

5. Letang E, Müller MC, Ntamatungiro AJ, et al. Cryptococcal antigenemia in immunocompromised human immunodeficiency virus patients in rural Tanzania: a preventable cause of early mortality. Open Forum Infect Dis 2015; 2:ofv046.

6. Chiller T. Making a difference with point-of-care diagnostics. Plenary Session presented at: ASLM; 2014 Dec 3; Cape Town.

7. Department of Health, South Africa. National consolidated guidelines for the prevention of mother-to-child transmission of HIV (PMTCT) and the management of HIV in children, adolescents and adults. Pretoria, South Africa: Department of Health, 2015.

8. Meyer AC, Kendi CK, Penner JA, et al. The impact of routine cryptococcal antigen screening on survival among HIV-infected individuals with advanced immunosuppression in Kenya. Trop Med Int Health 2013; 18:495-503.

9. Butler EK, Boulware DR, Bohjanen PR, Meya DB. Long term 5-year survival of persons with cryptococcal meningitis or asymptomatic subclinical antigenemia in Uganda. PLoS ONE 2012; 7:e51291.

10. Wong EB, Omar T, Setlhako GJ, et al. Causes of death on antiretroviral therapy: a post-mortem study from South Africa. PLoS ONE 2012; 7:e47542.

11. Karat AS, Omar T, von Gottberg A, et al. Autopsy prevalence of tuberculosis and other potentially treatable infections among adults with advanced HIV enrolled in out-patient care in South Africa. PLoS ONE 2016; 11:e0166158.

12. Omar T, Variava E, Moroe E, et al. Undiagnosed TB in adults dying at home from natural causes in a high TB burden setting: a post-mortem study. Int J Tuberc Lung Dis 2015; 19:1320-5.

13. Kowalska JD, Friis-Møller N, Kirk O, et al; CoDe Working Group; D:A:D Study Group. The Coding Causes of Death in HIV (CoDe) project: initial results and evaluation of methodology. Epidemiology 2011; 22:516-23.

14. Bicanic T, Meintjes G, Wood R, et al. Fungal burden, early fungicidal activity and outcome in cryptococcal meningitis in antiretroviral-naive or antiretroviralexperienced patients treated with amphotericin B or fluconazole. Clin Infect Dis 2007; 45:76-80. 
15. Dromer F, Bernede-Bauduin C, Guillemot D, Lortholary O; for the French Cryptococcosis Study Group. Major role for amphotericin B-flucytosine combination in severe cryptococcosis. PLoS ONE 2008; 3:e2870.

16. Jackson AT, Nussbaum JC, Phulusa J, et al. A phase II randomized controlled trial adding oral flucytosine to high-dose fluconazole, with short-course amphotericin B, for cryptococcal meningitis. AIDS 2012; 26:1363-70.

17. Nussbaum JC, Jackson A, Namarika D, et al. Combination flucytosine and highdose fluconazole compared with fluconazole monotherapy for the treatment of cryptococcal meningitis: a randomized trial in Malawi. Clin Infect Dis 2010; 50:338-44.

18. Sriruttan C, Wake R, Rukasha I, et al. Comparison of a novel semi-quantitative prototype and a commercial lateral flow assay for detection of cryptococcal antigen from thawed whole blood samples [Abstract 0140]. In: Fungal diagnostics. 26th European Congress of Clinical Microbiology and Infectious Diseases (ECCMID), Amsterdam, 2016.

19. Temfack E, Kouanfack C, Mossiang L, et al. Cryptococcal antigen screening in asymptomatic HIV-infected antiretroviral naïve patients in Cameroon and evaluation of the new semi-quantitative biosynex CryptoPS test. Front Microbiol 2018; 9:409.

20. Meya DB, Kiragga AN, Nalintya E, et al. Reflexive laboratory-based cryptococcal antigen screening and preemptive fluconazole therapy for cryptococcal antigenemia in HIV-infected individuals with CD4 $<100$ cells/ $\mu \mathrm{L}$ : a stepped-wedge, cluster-randomized trial. J Acquir Immune Defic Syndr 1999. 2019;80:182-9.

21. Rajasingham R, Wake RM, Beyene T, Katende A, Letang E, Boulware DR. Cryptococcal meningitis diagnostics and screening in the era of point-of-care laboratory testing. J Clin Microbiol 2019; 57:e01238-18.

22. Molloy SF, Kanyama C, Heyderman RS, et al; ACTA Trial Study Team. Antifungal combinations for treatment of cryptococcal meningitis in Africa. N Engl J Med 2018; 378:1004-17.

23. Jarvis JN, Leeme TB, Molefi M, et al. Short course high-dose liposomal amphotericin B for HIV-associated cryptococcal meningitis: a phase-II randomized controlled trial. Clin Infect Dis 2018

24. Lawrence DS, Youssouf N, Molloy SF, et al. AMBIsome Therapy Induction OptimisatioN (AMBITION): high dose ambisome for cryptococcal meningitis induction therapy in sub-Saharan Africa: study protocol for a phase 3 randomised controlled non-inferiority trial. Trials 2018; 19:649.

25. Larson B, Schnippel K, Ndibongo B, Long L, Fox MP, Rosen S. How to estimate the cost of point-of-care CD4 testing in program settings: an example using the Alere Pima ${ }^{\text {TM }}$ analyzer in South Africa. PLoS ONE 2012; 7:e35444.

26. Jani IV, Sitoe NE, Alfai ER, et al. Effect of point-of-care CD4 cell count tests on retention of patients and rates of antiretroviral therapy initiation in primary health clinics: an observational cohort study. Lancet 2011; 378:1572-9.

27. Patten GE, Wilkinson L, Conradie K, et al. Impact on ART initiation of pointof-care CD4 testing at HIV diagnosis among HIV-positive youth in Khayelitsha, South Africa. J Int AIDS Soc 2013; 16:18518.

28. Wake R, Glencross DK, Sriruttan C, Harrison TS, Govender NP. Cryptococcal antigen screening in HIV-infected adults-let's get straight to the point-of-care. AIDS 2016; 30:339-42.

29. Wake RM, Jarvis JN, Harrison TS, Govender NP. Brief report: point of care cryptococcal antigen screening: pipetting finger-prick blood improves performance of immunomycologics lateral flow assay. J Acquir Immune Defic Syndr 2018; 78:574-8.

30. Jarvis JN, Harrison TS, Corbett EL, Wood R, Lawn SD. Is HIV-associated tuberculosis a risk factor for the development of cryptococcal disease? AIDS 2010; 24:612-4.

31. Burton JL, Rutty GN, eds. The hospital autopsy: a manual of fundamental autopsy practice. 3rd ed. London: Hodder Arnold, 2010:352.

32. Jarvis JN, Harrison TS. Pulmonary cryptococcosis. Semin Respir Crit Care Med 2008; 29:141-50.

33. Driver JA, Saunders CA, Heinze-Lacey B, Sugar AM. Cryptococcal pneumonia in AIDS: is cryptococcal meningitis preceded by clinically recognizable pneumonia? J Acquir Immune Defic Syndr Hum Retrovirol 1995; 9:168-71.

34. Wong ML, Back P, Candy G, Nelson G, Murray J. Cryptococcal pneumonia in African miners at autopsy. Int J Tuberc Lung Dis 2007; 11:528-33.

35. Harris JR, Lindsley MD, Henchaichon S, et al. High prevalence of cryptococcal infection among HIV-infected patients hospitalized with pneumonia in Thailand. Clin Infect Dis 2012; 54:e43-50. 